\title{
A Look Into the 4G Crystal Ball
}

\author{
Simone Frattasi ${ }^{1}$, Frank H.P. Fitzek ${ }^{1}$, Ramjee Prasad ${ }^{1}$ \\ Center for TeleInFrastruktur (CTIF), Aalborg University, \\ Niels Jernes Vej 12, 9220, Aalborg, Denmark \\ $\{s f|f f|$ prasad $\} @ k o m . a a u . d k$
}

\begin{abstract}
The time for reflections and visions about the Fourth Generation of Wireless Mobile Communication Systems (4G) is getting closer to the $\mathrm{X}$ hour, therefore the research community has to finally declare how $4 \mathrm{G}$ will really look like. Besides the no-boundaries visions, in this paper we have a look into the $4 \mathrm{G}$ crystal ball, attempting to define the forthcoming system fusing both the user and the technology perspectives.
\end{abstract}

\section{Introduction}

The major reason for the "failure" of the Third Generation of Wireless Mobile Communication Systems (3G) has been the tremendous worldwide downturn in the economy that lead to a starvation of new investments and hence to delays in technological development, service roll out, etc. In some countries with high penetration of mobile services, this was also combined with a reward policy for releasing the spectrum usage rights that did not favor the investments in the International Mobile Telecommunications 2000 (IMT-2000) infrastructure. However, besides these difficulties, the evolution from the Second Generation (2G) towards $3 \mathrm{G}$ has not brought any substantial new service for the customer, leaving the business model largely unchanged. The well known services plus some additional ones are provided, but they may not be enough to encourage the customers to change their equipment. The lack of innovative and appealing services was encountered too late by the $3 G$ Partnership Project (3GPP). In the latest standards, an attempt was made to incorporate some advanced services into the 3GPP architecture such as the Multimedia Broadcast and Multicast Service Center (MBMS) in combination with the IP Multimedia System (IMS). Nevertheless, these smaller improvements were made without the possibility to adjust the access technology properly. Ultimately, it has to be underlined that the limited success of the new technology has also depended upon the cultural and social settings in which the new system has been deployed. Indeed, 3G has been more accepted in Asian countries than in Europe: in Japan, the teenagers share videos and books on their mobile phones in public places, whereas in Europe they experience the same exchange indoor, in their own rooms with their own TV-sets or computers.

The difficulties and the technical limitations of $3 \mathrm{G}[1]$, as well as the emergence of new mobile broadband technologies on the market, have brought universities and industries to a throughout reflection on the Fourth Generation (4G). 
The latter is expected to have a highly eclectic structure, where many devices, networks and protocols interact in a complex way to support the increasing user demands. Basically, many prophetic visions have appeared in literature [2], presenting the forthcoming generation as the ultimate boundary of the wireless mobile communication without any limit in its potential, but practically not giving any designing rule and thus any definition of it. Recently, a first attempt has been done within the framework of the Joint Advanced Development Enabling $4 \mathrm{G}$ (JADE) project ${ }^{1}$, where a pragmatic methodology [3], centered on a user-centric approach, has lead to the definition of the key features and the technological step-up to be undertaken in 4G [4]. This methodology answers critically to the MAGIC view of NTT DoCoMo [5] as well as to the somehow cloudy one of the European Community (EC) of connecting 'everything with everything' [6]: the technological possibilities derived from the heterogeneity of terminals and networks are tremendous if - and only if - they meet the customers' needs and requirements. Indeed, since the ultimate goal of the technology is for communication requirement of human beings and the service is, to some extant, exhibitions of human requirement for communication, the trend of the service provision will definitely impose its influence on the underlying architecture design [7]. This methodology is hence strictly oriented towards a use of the technology for better life conditions and fair societies, where the inner logic of the technological evolution is coordinated with the societal one.

In line with [3] and [4], "4G will be a convergence platform providing clear advantages in terms of coverage, bandwidth, and power consumption. Moreover, it will offer a variety of new heterogeneous services, from pop-up advertisements to location-based and interactive or on-demand ones - so called IP datacasting. All these characteristics will be supported by multi-mode / reconfigurable devices and the implementation of interworking ones". In order to achieve the previous outlined goals, in this paper we motivate the cellular controlled shortrange communication architecture adopted in JADE and consequently extend the framework developed in [3] and [4] - the "User-Centric" System - introducing the issue of cooperation. In particular, we show an innovative cooperative geolocation scheme supported by such an architecture that combining long- and short-range location information enhance the location estimation accuracy with respect to the actual stand-alone cellular solutions. This scheme could hence support in the future Cooperative Location Based Services (CLBSs). Finally, an insight on the social dimension related to cooperative services in general is also discussed at the end of the paper.

The rest of the article is structured as follows: Section 2 describes the JADE system architecture; Section 3 presents the "Cooperative-User-Centric" System as the extension of the "User-Centric" System; Section 4 illustrates the proposed Cooperative Localization Scheme (CLS); and Section 5 discusses the social dimension related to the proposed framework. Finally, the concluding remarks are given in Section 6.

1 The JADE project is a cooperation between SAMSUNG Korea and the Center for TeleInFrastruktur (CTIF), Aalborg University. 


\section{JADE System Architecture}

The overall target is a cellular system that also supports short-range communications among the terminals. The rationale for introducing short-range communications is mainly due to two arguments: 1) The need to support peer-to-peer (P2P) high-speed wireless links between the terminals; 2) The need to enhance the communication between a terminal and the Base Station (BS) by fostering cooperative communication protocols among spatially proximate devices. The communication enhancement primarily refers to a higher link reliability, a larger coverage, a higher spectral efficiency and a lower power consumption thanks to the use of exclusive cooperative stations (e.g., Relay Stations (RSs) deployed by operators) or short-range communications among different mobile terminals.

The cellular system will be synchronous and a tight control will be imposed by the BS over the short-range communications among the devices associated with it. The wireless terminals will use the same air interface for long- and shortrange communications. The expression "same air interface" means that the basic set of access technologies should be the same for links with the BS as well as P2P links. However, the access technologies should be tunable, such that a terminal can seamlessly adapt the transmission format to the cellular links and the shortrange $\mathrm{P} 2 \mathrm{P}$ ones, respectively. As an illustration, the same air interface implies that some transmission formats applied when a terminal transmits to the BS can be received also by another terminal. Nevertheless, the BS will apply more complex receiver algorithms and will thus be able to extract more data from the same transmitted message.

\subsection{Cooperative Communication}

Recently, much research effort has been put to understand and utilize the benefit of cooperative behavior in wireless networks [9]. The concept of cooperation introduces a new form of diversity where the terminals are less susceptible to the channel variations and shadowing effects. This results in an increased reliability of the communication and the extension of the coverage. Furthermore, whereas in voice networks the resources are dedicated for each user separately, in cellular controlled short-range data networks it is possible to group the users in clusters with the following advantages: a) Only the Cluster Head $(\mathrm{CH})$ needs to have a dedicated channel to the BS, while the other terminals can communicate by using unlicensed bands, thus more bandwidth is not required; b) Due to the short range of the transmissions performed by the terminals to the $\mathrm{CH}$, it is possible to reduce their power consumption and hence prolong their battery life.

In order to achieve the previous outlined goals, the work is mostly focused on the design of different cooperative mechanisms in the following layers of the protocol stack:

- Physical (PHY) layer. The research in PHY-layer cooperation is in a mere infancy, but it has already promised a great potential. Cooperative diversity protocols exploiting the feature of wireless broadcast medium have shown 
potential to achieve similar effect to conventional Multiple Input Multiple Output (MIMO) transmissions. However, theoretical analysis of capacity increase brought by cooperative behavior has been the main topic in the literature [10], while there have been few works on practical protocol designs to achieve the high diversity gain. This research area focuses on the design of coding, modulation, receiver algorithms and forwarding mechanisms such as Amplify and Forward (AF) and Decode and Forward (DF) in order to achieve high diversity gain in practical cooperative scenarios.

- Medium Access Control (MAC) layer. An important issue to be addressed is the formation of the cooperating group for different targets (coverage enhancement, energy consumption reduction, etc.), taking account of the interterminal channel conditions and the spatial distribution of the terminals. The design of protocols such as radio resource management and handoff has to be also addressed to coordinate transmissions between short-range links (links within cooperative groups) and long-range link (links between BS and terminals) so that the most appropriate links can be used by terminals with the least interference conditions.

\section{A "Cooperative-User-Centric" System}

The discussion about the system architecture adopted in JADE closes the circle of the methodological approach described in [3], fusing the user with the technological perspective, and addressing the technical step-up illustrated in [4]. In particular, the introduction of cooperation affects the "User-Centric" System, which in this section is extended to the "Cooperative-User-Centric" System.

Although we are used to think that the stars come as individuals because that's how our own Sun appears, this is not the norm. The evidence, instead, is that more than $85 \%$ of them are parts of multiple star systems, where the stars member revolve around a common center of mass under the influence of their mutual gravitational force. The most common multiple star system includes two stars and it is called binary star system. In some binary star systems, called close binaries, the stars are so close together that they can transfer matter to each other and change the way they look and evolve.

People, like stars, are seldom found in isolation. Therefore, along with the new architecture proposed in Section 2, we can represent $4 \mathrm{G}$ as a "CooperativeUser-Centric" System, where each user, according to [3] and [4], comprises its own planets (see Figure 1). Moreover, as multiple star systems when the stars are getting closer to each other raising up their velocity accordingly to their increasing mutual gravitational force (violet curve in Figure 1), the user cooperativity and consequently the services' performance obtained by clustering the users can be considerably enhanced (e.g., as shown in [8], the users' cooperation can lead to better services at lower prices). In the next section, a practical example of performance enhancement is described. 


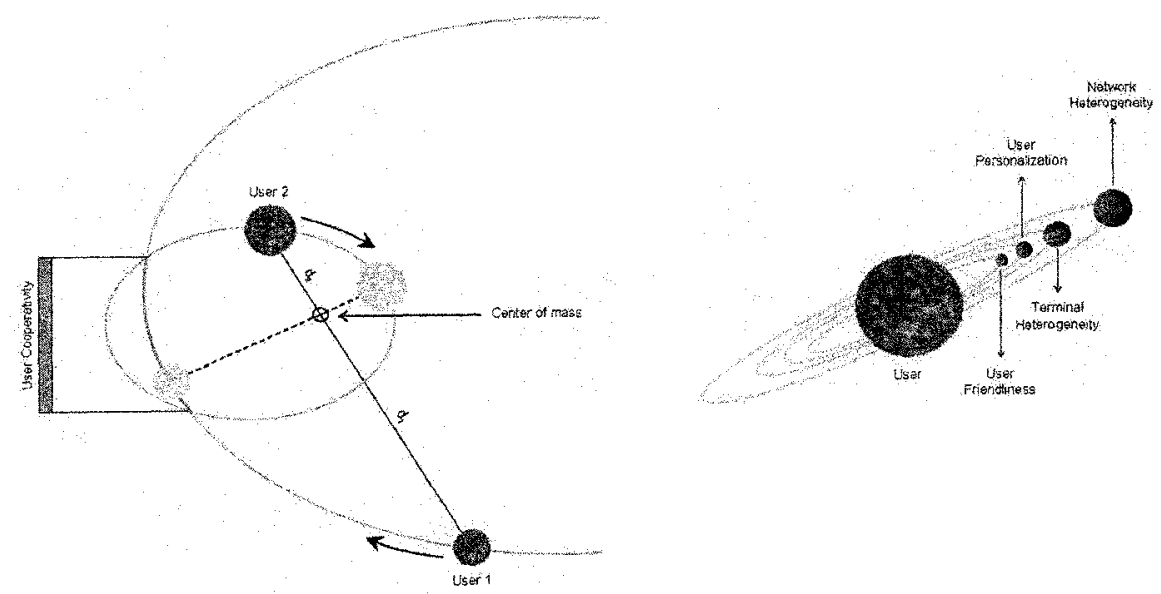

Fig. 1. The "Cooperative-User-Centric" System.

\section{Cooperative Localization}

Geolocation, or location estimation in terms of geographic coordinates of a $\mathrm{Mo}$ bile Station (MS) with respect to a reference point in a wireless system, has gained considerable attention over the past decade, especially since the Federal Communication Commission (FCC) passed a mandate requiring cellular providers to generate location estimates for Enhanced-911 (E-911) services with an accuracy of 100 meters for $67 \%$ of the cases [11]. This has boosted the research in the field of wireless location as an important public safety feature, which can also add many other potential applications to the future cellular systems [12].

In this section, we propose an innovative geolocation scheme that combines long- and short-range location information, respectively retrieved by mean of a Hybrid Time Of Arrival/Angle Of Arrival (HTA) technique in cellular networks and TOA technique in short-range networks, in order to enhance the location estimation accuracy with respect to the actual stand-alone cellular solutions. To the best of our knowledge, most of the existing studies in the literature tend to treat these positioning techniques separately for cellular and short-range networks. In the CLS, we practically suppose that the cellular system has supervised the formation of a short-range cluster and that the $\mathrm{CH}$ has relayed back all the measures of TOA corresponding to the measurements of relative distance obtained by each MS from the other members of the cooperative group ${ }^{2}$ (see Figure 2). The TOA measures are then exploited in the location algorithm in order to weight the HTA measures associated to the users belonging to the cluster.

\footnotetext{
${ }^{2}$ The CH sends the data to the nearest BS thanks to a possible power control procedure exploited during the clusters' initialization phase.
} 


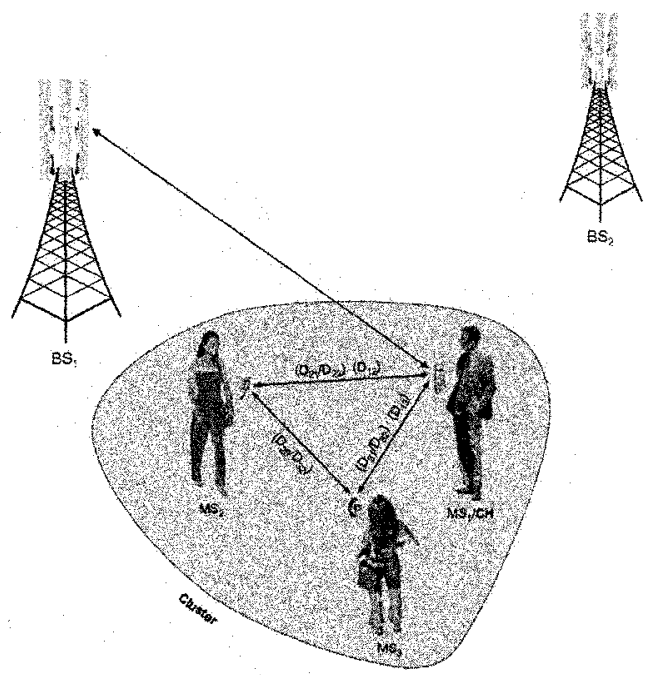

Fig. 2. Reference scenario.

System level simulations have been carried out developing a simulator in MATLAB. We have taken into consideration an urban-like scenario (micro cells of diameters around $2000 \mathrm{~m}$ ), where a group of $1 \leq M \leq 8 \mathrm{MSs}$ is simultaneously in the range of $3 \leq N \leq 7 \mathrm{BSs}$, where the $\mathrm{CH}$ is placed in the center of the cluster at a minimum distance of $100 \mathrm{~m}$ to the serving BS, being BS_1, and the other MSs are uniformly generated around it at a maximum distance of $100 \mathrm{~m}$. The BSs are positioned in a two-dimensional plane with coordinates BS_1 $(0,0)$, BS_2(1732,1000), BS_3(1732,-1000), BS_4 $(0,-2000)$, BS_5($1732,-1000)$, BS_6(-1732,1000) and BS_7 $(0,2000)$. We suppose that the speed of the users is very low and thus that, during the number of iterations considered for the location estimation, there is no relative movement between the cluster and the BSs, and within the cluster itself. Finally. we assume that the measurements are made on signals propagating via line-of-sight (LOS) paths; therefore, the estimation errors are small and primarily due to equipment measurement errors. The latter have traditionally been assumed to be normally distributed with zero mean and a small variance, respectively $c \sigma_{t}=30 \mathrm{~m}$ (the variances of the TOA measurement errors associated with different BSs are set as identical) and $\sigma_{\alpha}=1 \mathrm{deg}$. In particular, since each MS is carrying out a calculation on each short-range link, the measured distance between two MSs becomes more reliable and thus we set $c \sigma_{t_{\text {rel }}}=3 \mathrm{~m}$. The values of the parameters used in the simulations are summarized in Table 1.

The performances of the proposed scheme are presented in the form of $\mathrm{Cu}$ mulative Distribution Functions (CDFs) of the average location error, i.e., the estimation error resultant from the mean of the individual estimation errors, which has been computed based on 1000 independent runs. 
Table 1. Simulation parameters.

\begin{tabular}{l|r}
\hline PARAMETERS & VALUES \\
\hline Number of BSs & $1-7$ \\
Number of MSs & $1-8$ \\
Distance BS/CH & $100-900 \mathrm{~m}$ \\
Distance MS/CH & $1-100 \mathrm{~m}$ \\
TOA error (long-range) & $\mathrm{N}\left(0, \mathrm{c} \sigma_{t}=30\right)$ \\
TOA error (short-range) & $\mathrm{N}\left(0, c \sigma_{t_{r e l}}=3\right)$ \\
AOA error & $\mathrm{N}\left(0, \sigma_{\alpha}=1 \mathrm{deg}\right)$ \\
\hline
\end{tabular}

Figure 3 shows the improvements introduced by the proposed scheme with respect to a stand-alone HTA due to the users' cooperation and the estimation of their relative distances. Considering three BSs, for example, and estimating the location of the users only with the HTA technique, the distribution of the average location error has a mean $\mu=32.68 \mathrm{~m}$, whereas, in case of eight cooperative users, $\mu$ drops to $22.69 \mathrm{~m}$. This is due to the fact that the retrieved location estimates represent a certain configuration in the space, which has to respect the geometrical constrains of a polygonal structure (e.g., octagonal in case of eight users) based on the knowledge of the relative distances. As a consequence, the more cooperative users join the cluster the more binds the selected solution has to respect and the higher the accuracy is.
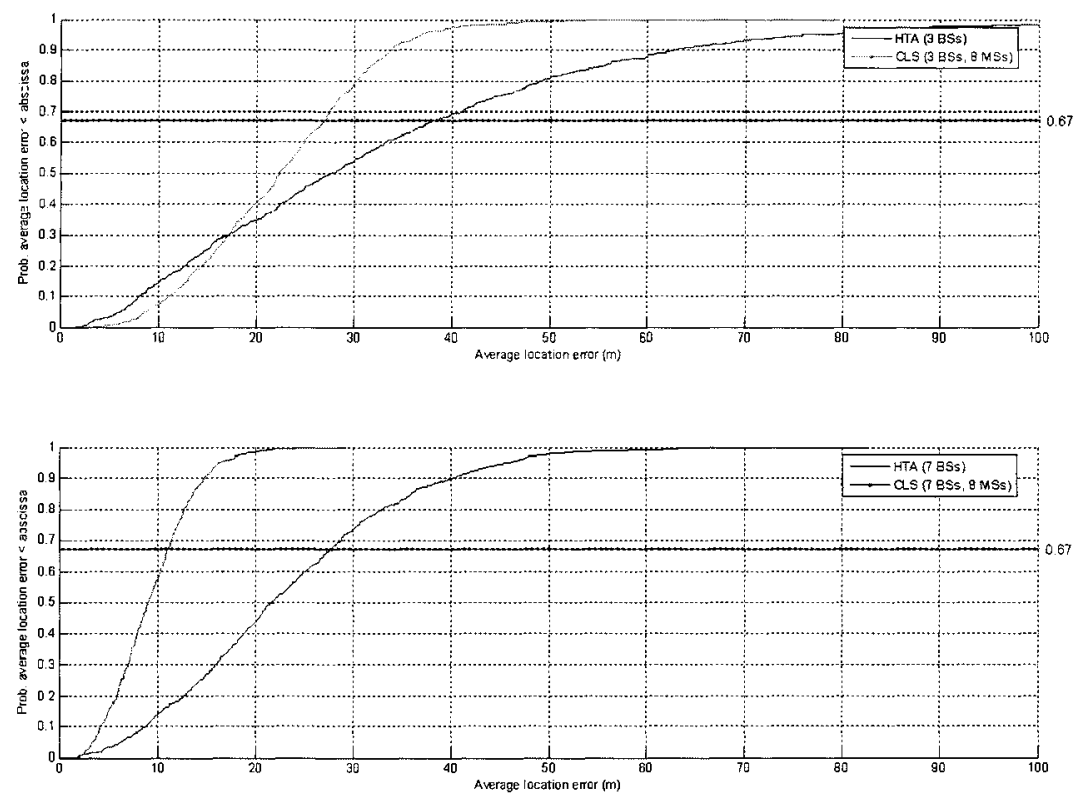

Fig. 3. Performance comparison between HTA and CLS. 
The simulation results show that the CLS increases the location estimation accuracy with respect to a stand-alone HTA solution. Furthermore, when $N \geq 3$ BSs are available for location purpose the average location error achieves values comparable to the Global Positioning System (GPS). The proposed scheme hence demonstrates that the synergy between cellular and short-range communication systems can represent a valid architecture for $4 \mathrm{G}$.

\section{The Social Dimension of Cooperative Services}

This section gives an insight on the potential social impact of cooperative services. The latter are mainly based upon three fundamental phenomena of social order:

1. Group building. Social groups on different levels (such as teenage groups, organized groups of football players, loosely organized groups of neighbors, which are relative stable over the time, or loosely organized and only for a short time stable internet groups) are the intermediaries in societies. Groups differ concerning what they have in common: spatial proximity (neighbors), interests (internet groups, business groups), age (teenage groups) and so forth. They are more or less durable over the time and more or less organized. Although the service provision is performed at the technical level, on the social level new groups might be established to lower the costs and offer a better quality of the services.

2. Network. As Manuel Castells outlined [13], fuzzy, spontaneous networks are increasing in importance. Cooperative services make intelligent use of these decentralized network of terminals, where the necessary but limited hierarchy is evolving out of the given situation and the decision concerning the master-terminal is drawn on the background of technical means and conditions seamless to the users. These new type of networks may be hence coined situational hierarchy and indicate the increasing relevance of new types of time-limited and functional networks. As a consequence, the success of cooperative services will depend not only on the potential resulting performance but also on the trust the customers have in the capability of the network to define a master-terminal that will be really able to coordinate.

3. Cooperation/sharing. Cooperation in the society is usually defined as a coordinated effort to reach mutual goals. The different reasons at the base of this coordination are: traditions, habits, emotions (like compassion), instrumental rational considerations (like efficiency, utility) or more normative rational ones (what one ought to do according a given social or moral norm). It depends very much on the context and the behavioral setting as well as on the motivation in the background and thus in the stability of the cooperation. Although the common sense makes us believe that the best motivation for cooperation is a personal good outcome or result, the concept of the self-interested actor that tries to maximize his own interests and profits is not always valid. In the cooperative use of the mobile phone, for example, 
the rational interest of lowering costs and receiving a better quality of the services lies clearly in the realm of maximizing the profit. Nevertheless, also to become member of a group and to share not only material but symbolic resources (e.g., joint values, prestige, friendship, etc.), could be a strong motivation for utilizing such cooperative services. Indeed, we can imagine groups that would like to make clever use of them in order to watch videos or share other files that are usually more expensive. Therefore, ad-hoc communities based on agreements about what to watch together are then possible to set up. These services hence increase the cooperative behavior and empowers the consumer to make clever use of them. In general, the possibility for customers to deal in a creative manner with the technical possibilities is of great importance for the services' acceptance. In a way, the user terminal is not any more a bare medium to transfer information, but a social medium that helps to build groups and friendships.

\section{Conclusions}

Even though in the research community there is still uncertainty regarding the final silhouette of $4 \mathrm{G}$, the objectives are quite clear and indeed the motto of the forthcoming system(s) is: " $4 \mathrm{G}$ has to be cheaper and better than $3 \mathrm{G}$ ". In this paper, as a result of a joint user-technology analysis, we have defined $4 \mathrm{G}$ according to the JADE project. The new system concept - the "CooperativeUser-Centric" System - has shown to potentially achieve the goals underlined in the $4 \mathrm{G}$ motto.

\section{Acknowledgements}

This work has been supported by Samsung Electronics, Co., LTD, Korea.

\section{References}

1. L. Zhen, Z. Wenan, S. Junde, H. Chunping, "Consideration and Research Issues for the Future Generation of Mobile Communication", in Proceedings of the 15th Canadian Conference on Electrical and Computer Engineering (CCECE), IEEE, vol. 3, pp. 1276-1281, Winnipeg, Manitoba, Canada, May 12-15, 2002.

2. E. Bohlin, S. Lindmark, J. Bjrkdahl, A. Weber, B. Wingert, P. Ballon, "The Future of Mobile Communications in the EU: Assessing the Potential of 4G", ESTO Publications, February, 2004.

3. S. Frattasi, H. Fathi, F.H.P. Fitzek, M. Katz, R. Prasad, "A Pragmatic Methodology to Design 4G: From the User to the Technology", in Proceedings of the 5th International Conference on Networking (ICN), IEEE, Reunion Island, France, April 17-21, 2005.

4. S. Frattasi, H. Fathi, F.H.P. Fitzek, K. Chung, R. Prasad, "4G: The User-Centric System", Mobile e-Conference (Me), Electronic Conference, August, 2004. 
5. K. Murota, NTT DoCoMo, "Mobile Communications Trends in Japan and DoCoMo's Activities Towards 21st Century", in Proceedings of the 4th ACTS Mobile Communications Summit, Sorrento, Italy, June 8-11, 1999.

6. J. M. Pereira, "Fourth Generation: Now, it is Personal", in Proceedings of the 11 International Symposium on Personal, Indoor and Mobile Radio Communications (PIMRC), IEEE, London, UK, September 18-21, 2000.

7. J. Yang, Z. Ping, "MUSE: A Vision for $4 G$ Service and Architecture", in Proceedings of the 60th Vehicular Technology Conference (VTC), IEEE, Los Angeles (CA), USA, September 26-29, 2004.

8. S. Frattasi, B. Can, F.H.P. Fitzek, R. Prasad, "Cooperative Services for $4 G$ ", in Proceedings of the 14th IST Mobile \& Wireless Communications Summit, Dresden, Germany, June 19-23, 2005.

9. A. Nosratinia, T. E. Hunter, A. Hedayat, "Cooperative Communication in Wireless Networks", Communications Magazine, IEEE, vol. 42, no. 10, pp. 74-80, October, 2004.

10. G. Li, H. Liu, "On the Capacity of Broadband Relay Networks", in Proceedings of the 38th Annual Asilomar Conference on Signals, Systems and Computers, Pacific Grove, CA, USA, November 7-10, 2004.

11. FCC, "FCC Acts to Promote Competition and Public Safety in Enhanced Wireless 911 Services", Washington, DC: WT Rep. 99-27, Sept. 15, 1999.

12. A.H. Sayed, N.R. Yousef, "Wireless Location", Wiley Encyclopedia of Telecommunications, J. Proakis, editor, John Wiley \& Sons, NY, 2003.

13. M. Castells, "The Information Age: Economy, Society and Culture", vol. 1-3, Blackwell, Oxford, 1998. 